\title{
Thermotropic Liquid Crystalline Polycarbonates VI. Synthesis and Properties of Fully Aromatic Liquid Crystalline Polycarbonates by Interfacial or Solution Polycondensation
}

\author{
Shih-Jieh Sun, Keh-Ying Hsu, and Teh-Chou Chang ${ }^{\dagger}$ \\ Department of Chemical Engineering, Chung Yuan Christian University, \\ Chung Li, Taiwan 32023, Republic of China
}

(Received May 7, 1996)

\begin{abstract}
Several series of fully aromatic liquid crystalline polycarbonates were synthesized by interfacial or solution polycondensation from triphosgene with aromatic diols, such as hydroquinone, 4,4'-biphenyldiol, 2,6-naphthalenediol, Bisphenol A, 4,4'-dihydroxydiphenyl sulfone, 4,4'-dihydroxydiphenylsulfide, 4,4'-dihydroxydiphenylmethane, 4,4'-dihydroxydiphenyl ether, methylhydroquinone, $t$-butylhydroquinone, phenylhydroquinone, and resorcinol, respectively. The structures and thermal properties of the synthesized polycarbonates were examined by FTIR spectroscopy, differential scanning calorimetry (DSC), thermal optical polarized microscopy, and thermogravimetric analysis (TGA). The effects of the structures of polycarbonates on liquid crystalline properties and thermal stability were investigated. The thermotropic LC properties of polycarbonates are strongly dependent on the structures and content of the bent units. The non-linearity of carbonate linkage can be compensated for by bent units in some case.

KEY WORDS Liquid Crystalline / Polycarbonate / Interfacial Polycondensation / Solution Polycondensation
\end{abstract}

Liquid crystalline polymers are a topic of intensive scientific investigation because of their unique thermal, rheological, and mechanical properties. A number of thermotropic liquid crystalline polymers, such as polyester, ${ }^{1-3}$ poly(ether-ester), ${ }^{4}$ poly(imide-ester), ${ }^{5-8}$ poly(amide-ester), ${ }^{9-11}$ polyazomethine, ${ }^{12}$ and polyurethane,$^{13,14}$ has been investigated. However, the carbonate group does not adopt a linear structure, and is considered not to contribute a liquid crystalline phase. Thermotropic liquid crystalline polycarbonates have only been reported in few papers. ${ }^{15-20}$ In our previous papers of this series, ${ }^{21,22}$ it was suggested that the carbonate did not severely affect the linear conformation but could reduce the melting transition temperature effectively.

Several synthetic methods for polycarbonates have been reported, such as (1) by the interfacial or solution polymerization of phosgene with diols, ${ }^{23}$ (2) interfacial or solution polymerization of phosgene substitutes, such as $N, N^{\prime}$-carbonyldiimidazole, trichloromethyl chloroformate, triphosgene, and bis(2,4,6-trichlorophenyl)carbonate with diols, ${ }^{24,25}$ (3) melt polymerization of diarylcarbonate with diols, ${ }^{21,22}$ (4) ring opening polymerization of cyclic oligomeric carbonates, ${ }^{26}$ and (5) solution polymerization of $\mathrm{CO}_{2}$, diols and dihalides. ${ }^{27}$ Commercial polycarbonates have been produced mainly by the phosgenation of diols. It is inconvenient to handle phosgene on the laboratory scale for polycarbonate synthesis. However, high-molecular-weight polycarbonate can be obtained using phosgene substitutes on the laboratory scale.

In this study, fully aromatic liquid crystalline polycarbonates were synthesized by the interfacial or solution polycondensation from triphosgene with aromatic diols. The effects of the structures of polycarbonates on liquid crystalline properties and thermal stability, and the liquid crystalline properties of polycarbonate containing the 2,6-naphthalene unit as a mesogenic group were investigated.

\section{EXPERIMENTAL}

\section{Material and Measurements}

Diols: 1,6-hexanediol (Merck Co.,), 1,10-decanediol (Merck Co.), hydroquinone (Merck Co.), 4,4'-biphenyldiol (Merck Co.), 2,6-naphthalenediol (Aldrich Co.), Bisphenol A (Merck Co.), 4,4'-dihydroxydiphenyl ether (Tokyo Kasei Co.), 4,4'-dihydroxydiphenyl sulfone (Merck Co.), 4,4'-dihydroxydiphenylsulfide (Merck Co.), 4,4'-dihydroxybenzophenone (Tokyo Kasei Co.), phenylhydroquinone (Merck Co.), and resorcinol (Merck Co.) were used as received. Triphosgene (Tokyo Kasei Co.) and benzyltriethylammonium chloride (Merck Co.) were used without further purification. Pyridine and $\mathrm{CH}_{2} \mathrm{Cl}_{2}$ were purified by distillation over $\mathrm{KOH}$ and $\mathrm{NaH}$, respectively, before use.

IR spectra from $4600 \mathrm{~cm}^{-1}$ to $400 \mathrm{~cm}^{-1}$ of the synthesized polymers in solid state were obtained by the $\mathrm{KBr}$ method on a JASCO FT/IR-70000. WAXD powder patterns were measured by a Rigaku D/MAX III, V XRD X-ray diffractometer at room temperature with $\mathrm{Cu}-K_{\alpha}$ radiation using $\mathrm{Ni}$-filter.

Visual observation of liquid crystalline transitions under cross polarized light was made using a Leitz Ortholux 2 POL BK polarizing microscope equipped with a Linkam THMS 600 heating stage. Thermal transition properties were characterized by a Du Pont 2000 DSC at a heating rate of $20^{\circ} \mathrm{Cmin}^{-1}$ under nitrogen atmosphere. Thermal stability data of polycarbonates were obtained from a Perkin Elmer TGA7 at a heating rate of $20^{\circ} \mathrm{C} \mathrm{min}^{-1}$ under nitrogen atmosphere.

\footnotetext{
${ }^{\dagger}$ To whom all correspondence should be addressed.
} 
Polymer Synthesis

A. Preparation of Bisphenol A-Polycarbonate (PC$B P A)$ by Interfacial Polycondensation

Method A (Run No. 1) Bisphenol A $(10 \mathrm{mmol})$, triphosgene (3.3 mmol), and benzyltriethylammonium chloride $(20 \mathrm{mg})$ were dispersed in dried dichloromethane $(40 \mathrm{ml})$. The mixture was added to $40 \mathrm{ml} 1 \mathrm{~N} \mathrm{NaOH}_{(\mathrm{aq})}$ immediately, and reacted at $0-10^{\circ} \mathrm{C}$ for $1 \mathrm{~h}$ with a high-speed mechanical stirrer. The whole mixture was poured into methanol. The obtained polymer was washed with methanol and dried at $85^{\circ} \mathrm{C}$ under vacuum for $12 \mathrm{~h}$. Polymers 2-4 were prepared by a similar procedure.

Method B (Run No. 5) Dichloromethane $(30 \mathrm{ml})$ was added to a solution of Bisphenol A $(10 \mathrm{mmol})$ and benzyltriethylammonium chloride $(20 \mathrm{mg})$ in $1 N \mathrm{NaOH}_{(\mathrm{aq})}$ $(40 \mathrm{ml})$. To this vigorously stirred solution, triphosgene $(3.5 \mathrm{mmol})$ in dried dichloromethane $(10 \mathrm{ml})$ was added dropwise and reacted at $0-10^{\circ} \mathrm{C}$ for $1 \mathrm{~h}$. The whole mixture was poured into methanol. The obtained polymer was washed with methanol and dried at $85^{\circ} \mathrm{C}$ under vacuum for $12 \mathrm{~h}$. Polymer 6 was prepared by a similar procedure.

Method C (Run No. 7) Bisphenol A (10 mmol), triphosgene $(3.0 \mathrm{mmol})$, and benzyltriethylammonium chloride $(20 \mathrm{mg})$ were dispersed in dried dichloromethane $(40 \mathrm{ml})$. The mixture was added to $40 \mathrm{ml} 1 N \mathrm{NaOH}_{(\mathrm{aq})}$ immediately, and reacted at $0-10^{\circ} \mathrm{C}$ for $15 \mathrm{~min}$ with a high-speed mechanical stirrer. Additional triphosgene $(0.5 \mathrm{mmol})$ in dichloromethane $(5 \mathrm{ml})$ was introduced dropwise. The whole mixture was reacted at $10^{\circ} \mathrm{C}$ for another $45 \mathrm{~min}$ and then poured into methanol. The obtained polymer was washed with methanol and dried at $85^{\circ} \mathrm{C}$ under vacuum for $12 \mathrm{~h}$. Polymers $8-9$, BP and HQ series polymers were prepared by a similar procedure.

B. Preparation of PC-BPA by solution polycondensation

Method $A$ (Run No. 1) Pyridine $(10 \mathrm{ml})$ was added dropwise to a solution of Bisphenol A $(10 \mathrm{mmol})$ and triphosgene $(3.3 \mathrm{mmol})$ in dichloromethane $(10 \mathrm{ml})$. The mixture was reacted at $5-10^{\circ} \mathrm{C}$ for $30 \mathrm{~min}$, at room temperature for $30 \mathrm{~min}$, and poured into methanol. The obtained polymer was washed with methanol and dried at $85^{\circ} \mathrm{C}$ under vacuum for $12 \mathrm{~h}$. Polymers $2-5$ were prepared similarly procedure.

Method B (Run No. 6) Triphosgene $(3.3 \mathrm{mmol})$ in dichloromethane $(10 \mathrm{ml})$ was added dropwise to a solubion of Bisphenol A $(10 \mathrm{mmol})$ in pyridine $(10 \mathrm{ml})$. The mixture was reacted at $5-10^{\circ} \mathrm{C}$ for $30 \mathrm{~min}$, then at the room temperature for another $30 \mathrm{~min}$, and poured into methanol. The obtained polymer was washed with methanol and dried at $85^{\circ} \mathrm{C}$ under vacuum for $12 \mathrm{~h}$. Polymers 7-10 and the Nap series (with naphthalene structure) were prepared by a similar procedure.

\section{RESULTS AND DISCUSSION}

\section{Polymer Synthesis}

The general synthetic routes and structures of polycarbonates are given in Scheme 1. Triphosgene was used as the acylation agent for the preparation of aromatic polycarbonates. Interfacial and solution polymerizations, were conducted for the preparation of

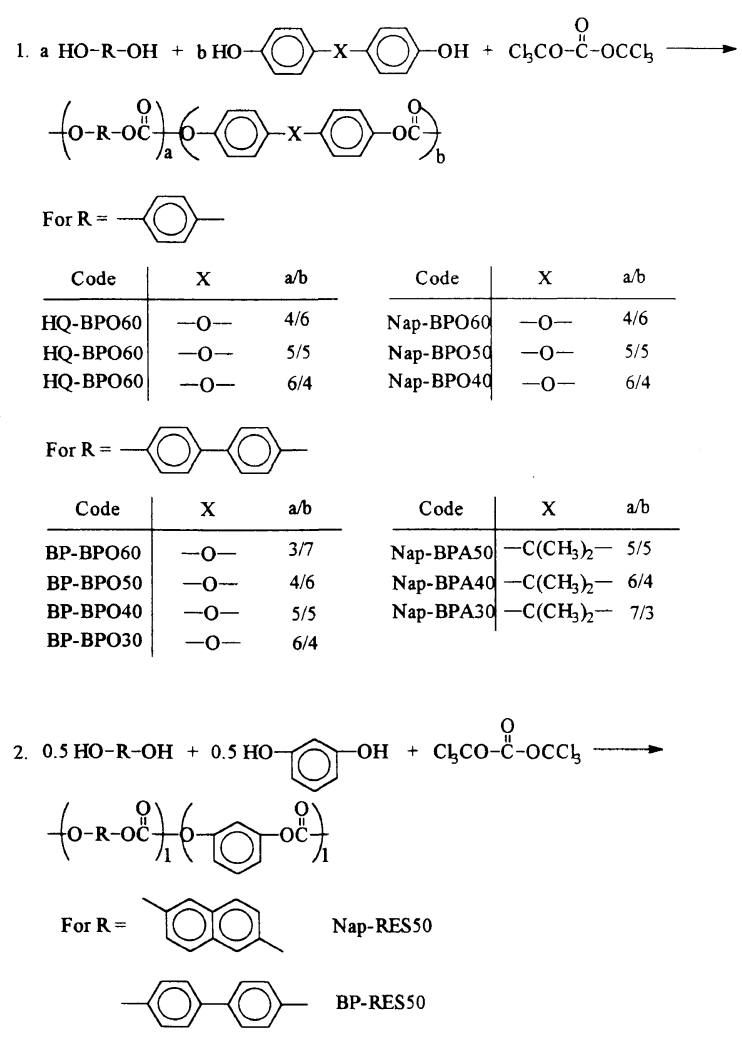

Scheme 1. Structures and general synthetic routes of polycarbonates.

Table I. Yields and inherent viscosities of PC-BPA prepared by interfacial polycondensation

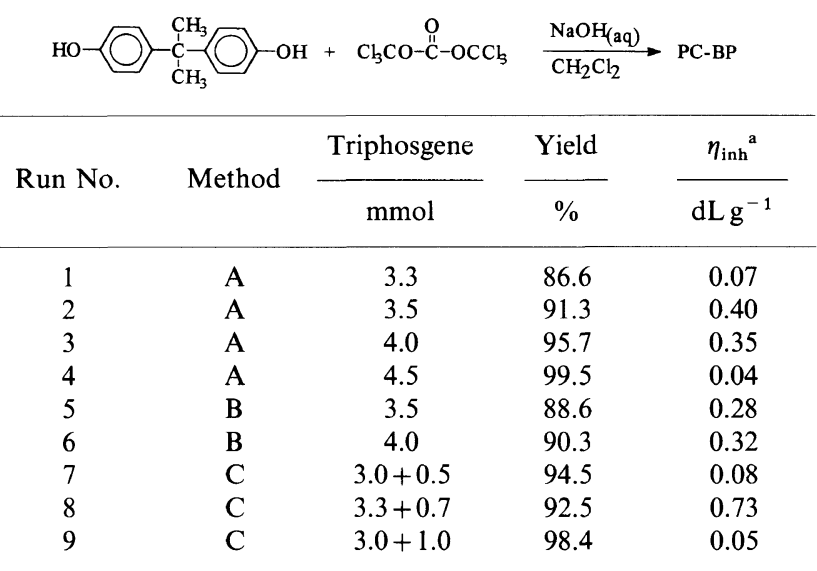

${ }^{\text {a }}$ Measured at $0.5 \mathrm{dL} \mathrm{g}^{-1}$ in DMF at $30^{\circ} \mathrm{C}$.

polycarbonates. The effects of the reaction of Bisphenol A with various amounts of triphosgene on yield and inherent viscosity by interfacial polymerization are listed in Table I. Polycarbonates obtained by method B showed lower molecular weight than by method A, possibly since the hydrolysis of triphosgene is very rapid in water. Run 8 , shown in Table I, a high-molecular-weight polycarbonate was done by the addition of excess $(0.7 \mathrm{mmol})$ triphosgene.

The effects of the reaction of Bisphenol A with various amounts of triphosgene on yield and inherent viscosity by solution polymerization were listed in Table II. Similar results were obtained to those of the interfacial polymerization and the inherent viscosity of the polycarbonate showed significant dependence on the amount of triphosgene. The yield of polycarbonate increased with 
Table II. Yields and inherent viscosities of PC-BPA prepared by solution polycondensation

$\mathrm{HO}-\mathrm{O}-\stackrel{\mathrm{C}}{\mathrm{C}}-\stackrel{\mathrm{C}}{\mathrm{C}}-\stackrel{\mathrm{O}}{\mathrm{C}}-\mathrm{OH}+\mathrm{Cl}_{3} \mathrm{CO}-\stackrel{\mathrm{C}}{\mathrm{C}}-\mathrm{OCCl}_{3} \stackrel{\text { Pyridine }}{\mathrm{TCE}}$ PC-BP

\begin{tabular}{|c|c|c|c|c|}
\hline \multirow{2}{*}{ Run No. } & \multirow{2}{*}{ Method } & Triphosgene & Yield & $\eta_{\mathrm{inh}}^{\mathrm{a}}$ \\
\hline & & $\mathrm{mmol}$ & $\%$ & $\mathrm{dLg}^{-1}$ \\
\hline 1 & A & 3.3 & 71.1 & 0.05 \\
\hline 2 & A & 3.5 & 81.0 & 0.32 \\
\hline 3 & A & 3.7 & 86.9 & 0.30 \\
\hline 4 & A & 4.0 & 92.9 & 0.39 \\
\hline 5 & A & 4.2 & 93.5 & 0.30 \\
\hline 6 & B & 3.3 & 60.5 & 0.04 \\
\hline 7 & B & 3.7 & 70.1 & 0.05 \\
\hline 8 & B & 3.8 & 80.8 & 0.25 \\
\hline 9 & B & 4.0 & 88.9 & 0.56 \\
\hline 10 & B & 4.2 & 92.3 & 0.42 \\
\hline 11 & B & 4.4 & 98.0 & 0.47 \\
\hline
\end{tabular}

${ }^{\text {a }}$ Measured at $0.5 \mathrm{dLg}^{-1}$ in DMF at $30^{\circ} \mathrm{C}$.

the amount of triphosgene. The inherent viscosity of the synthesized polycarbonates decreased with additional triphosgene $(>4.0 \mathrm{mmol})$. Viscous adducts were formed by adding pyridine to Bisphenol A/triphosgene/dichloromethane solution (method A), ${ }^{23}$ and only low-molecularweight polycarbonate was obtained. However, a relative high-molecular-weight polycarbonate was obtained by adding triphosgene to Bisphenol $\mathrm{A} /$ pyridine solution dropwise (method B).

In this study, HQ (hydroquinone), BP (4,4'-biphenyldiol), and Nap (2,6-naphthalenediol) series were synthesized as shown in Scheme 2. Polymers HQ and BP series were synthesized by interfacial polycondensation from HQ or BP with BPO (4,4'-dihydroxydiphenyl ether) or BPA (Bisphenol A) with triphosgene using benzyltri-

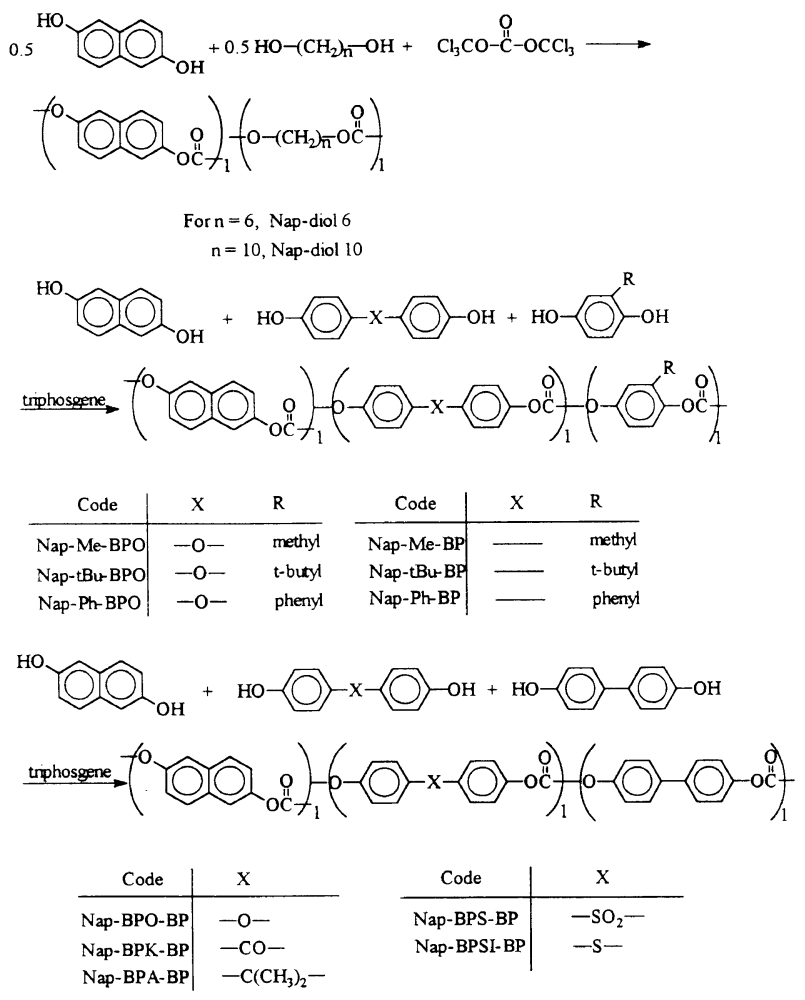

Scheme 2. Structures and general synthetic routes of polycarbonates. ethylammoniun chloride as a catalyst. Due to the good solubility of hydroquinone in water, only low-molecularweight polycarbonates were obtained for the HQ series. ${ }^{19}$ BP-series polycarbonates containing bisphenol (BPA or BPO) structures showed better solubility in the organic phase, thus a higher inherent viscosity of polymer could be obtained in the form of fibrous material. Gelation occurred during the polymerization of the BPO-unit rich polycarbonate, BP-BPO60 and BP-BPO70. Thus, only relatively low-molecular-weight polycarbonates were obtained.

Due to the good solubility of 2,6-naphthalenediol in water and poor solubility in dichloromethane, only low-molecular-weight polycarbonates were synthesized by interfacial polycondensation. Therefore, polycarbonates containing naphthalene structures were prepared using the solution polycondensation of triphosgene, 2,6-naphthalenediol and various aromatic diols using pyridine as the condensation agent and dichloromethane as the solvent. Binary Nap-series polycarbonates showed poor solubility in dichloromethane and precipitated during polymerization. Ternary polycarbonates prepared from phenylhydroquinone or bisphenols were obtained as fibrous material with relatively high molecular weights.

The BP-series polycarbonates, except for BP-BPA30, and Nap-series ternary polycarbonates dissolved in $N$-methyl-1-pyrrolidone. Not all the polycarbonates containing 2,6-naphthalene structures could dissolve in common organic solvent, but dissolve in phenol/1,1,2,2tf:trachloromethane $(6: 4)$ cosolvent by heating. The inherent viscosity of polymers was (listed in Tables III and IV) measured in phenol/1,1,2,2-tetrachloro-methane $(6: 4)$ cosolvent.

The structures of the resulting polycarbonates were characterized by FTIR spectroscopy. As shown in Figure 1, the FT-IR spectra of polymer BP-BPO50 contained strong absorption bands of the carbonate group at $1773 \mathrm{~cm}^{-1}$, phenylene group at $1597,1495 \mathrm{~cm}^{-1}$, and ether group at $1226,1164 \mathrm{~cm}^{-1}$, all characteristic for the aromatic polycarbonate system.

\section{Thermal Properties}

The thermal properties of the synthesized polymers evaluated by DSC, TGA measurements, and thermal optical polarized microscopic observation are summarized in Tables III and IV. DSC traces of most of the polycarbonates showed only a glass transition $\left(T_{\mathrm{g}}\right)$. This indicated that the polymers are an amorphous in character, as also confirmed by WAXD measurements. No liquid crystalline (LC) behavior was found for the HQ-series polycarbonates with various BPO content. This is reasonable since the aspect ratio of hydroquinone is not high enough. By using a higher aspect ratio monomer, BP, as the mesogenic unit, polymer BP-BPO60 with $60 \%$ BPO content showed LC behavior in the range of $226-332^{\circ} \mathrm{C}$. under a thermal optical polarized microscope. No LC properties were observed for polycarbonates synthesized from BP at various content of BPA, possibly due to the fact that BPA is a bent shape monomer with $s p^{3}$ structure and the rigidity of polymer chain is reduced by inserting BPA into the polymer main chain causing the absence of the LC properties. ${ }^{28}$ Figure 2 shows the DSC curves of polymer BP-BPO60, BP- 


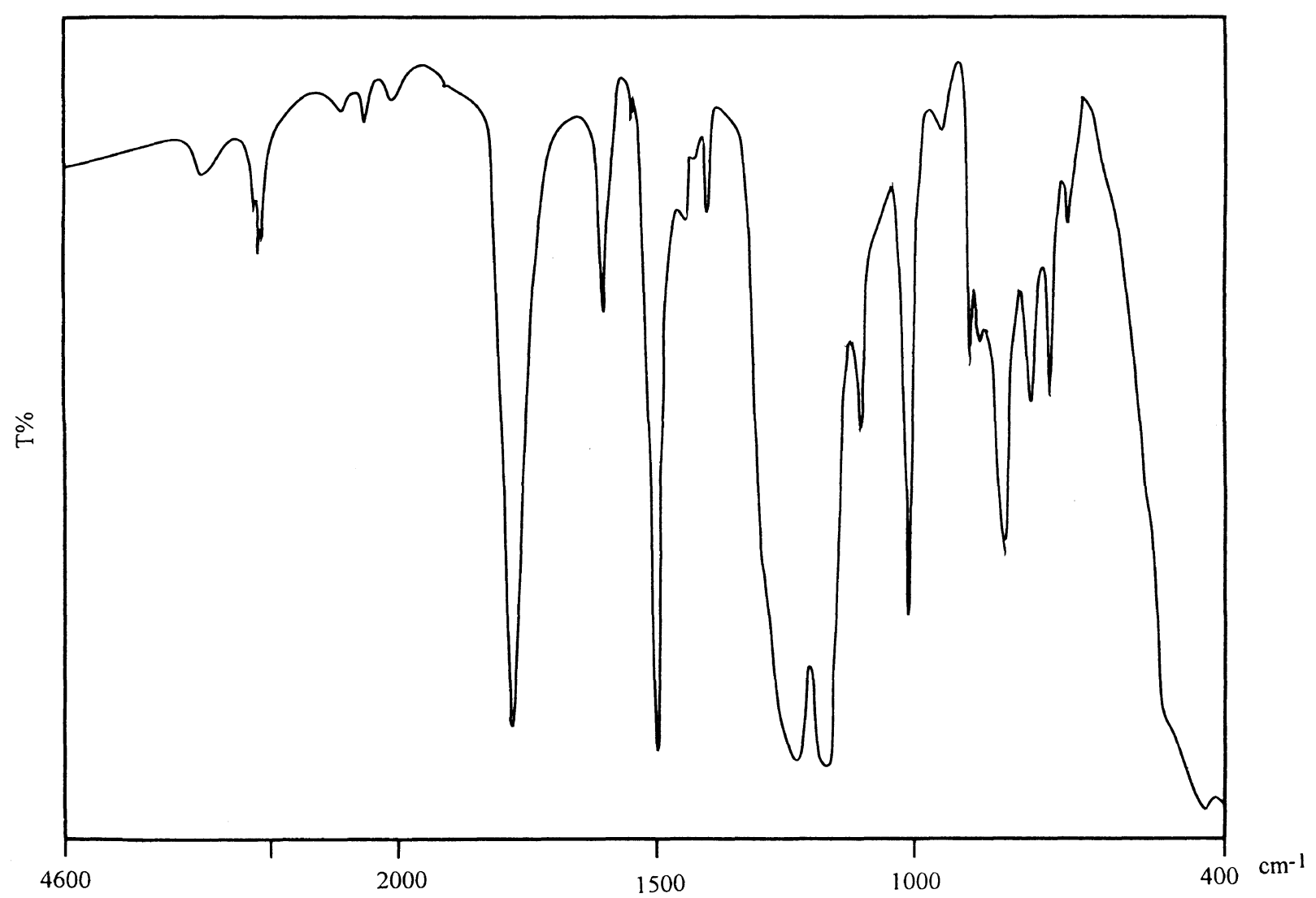

Figure 1. FT-IR spectra of Polymer BP-BPO60.

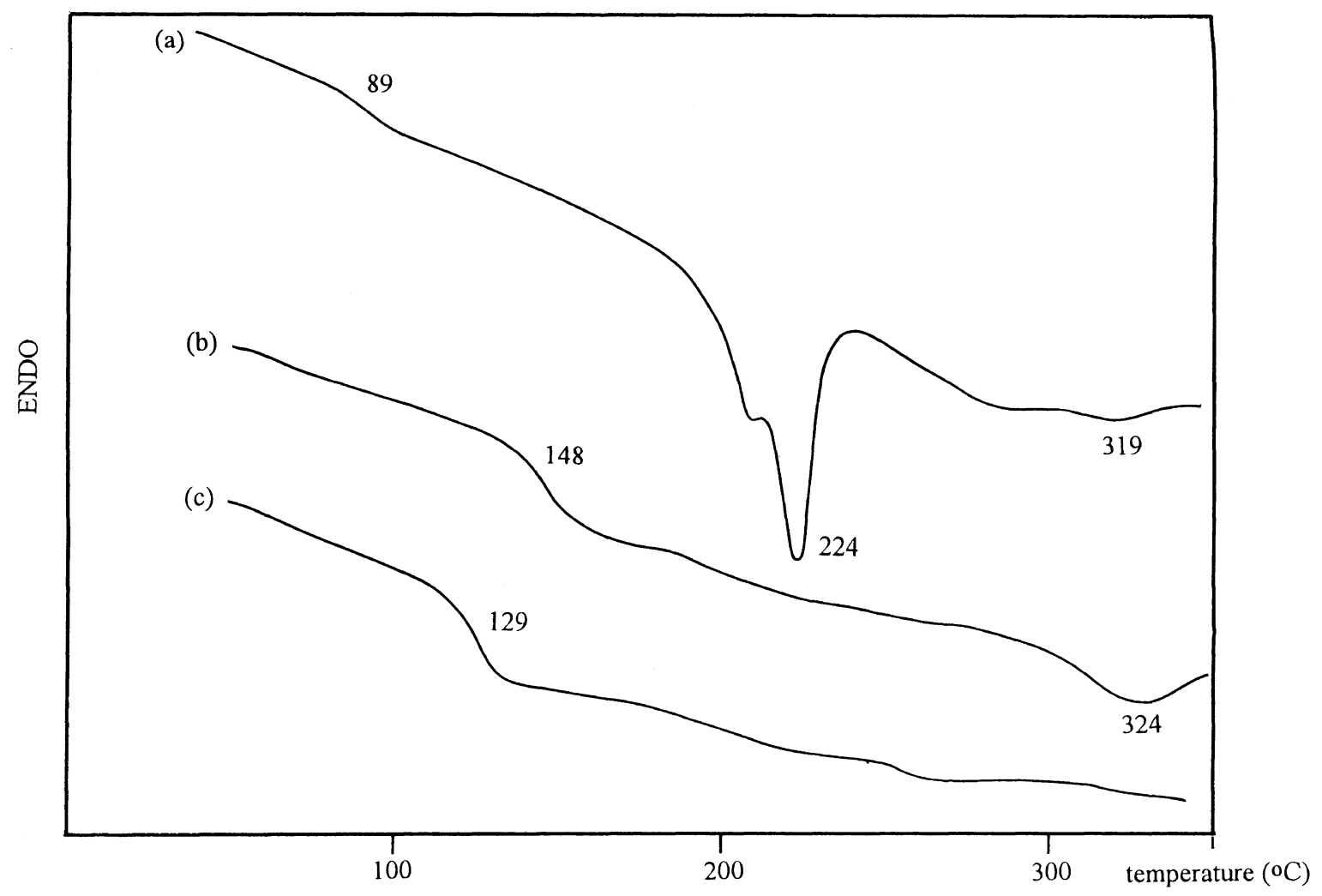

Figure 2. DSC measurements of (a) polymer BP-BPO60, (b) BP-BPA50, (c) HQ-BPO50, at a heating rate of $20^{\circ} \mathrm{Cmin}^{-1}$.

BPA50, and HQ-BPO50 at the second heating. Only a glass transition located at $129^{\circ} \mathrm{C}$ was detected for polymer HQ-BPO50. This indicates that polymer HQBPO50 is amorphous material at room temperature. The DSC curve of polymer BP-BPO60 showed a glass transi- tion $\left(T_{\mathrm{g}}\right)$ and two endotherms at $89^{\circ} \mathrm{C}, 224^{\circ} \mathrm{C}$, and $319^{\circ} \mathrm{C}$, respectively. As shown in Figure 3, a nematic melt was observed for polymer BP-BPO60 at $226-332^{\circ} \mathrm{C}$ by thermal optical polarized microscope. By introducing $70 \%$ content of BPO, BP-BPO70 lost it's LC properties. 
Thermotropic Liquid Crystalline Polycarbonates VI.

Table III. Properties of binary polycarbonates

\begin{tabular}{|c|c|c|c|c|c|c|c|c|c|c|}
\hline \multirow{2}{*}{ No. } & \multirow{2}{*}{$\frac{\text { Yield }}{\%}$} & \multirow{2}{*}{$\frac{\eta_{\text {inh }}{ }^{\mathrm{a}}}{\mathrm{dL} \mathrm{g}^{-1}}$} & \multirow{2}{*}{$T_{\mathrm{m}}^{\mathrm{b}}$} & \multirow{2}{*}{$T_{\mathrm{i}}^{\mathrm{b}}$} & \multicolumn{3}{|c|}{$\mathrm{DSC}^{\mathrm{c}}$} & \multicolumn{3}{|c|}{$\mathrm{TGA}^{\mathrm{e}}$} \\
\hline & & & & & $T_{\mathrm{g}}$ & $T_{\mathrm{m}}$ & $T_{\mathrm{i}}$ & $5 \%$ & $10 \%$ & $20 \%$ \\
\hline HQ-BPO50 & 76 & 0.36 & 219 & & 129 & & & 375 & 410 & 433 \\
\hline HQ-BPO40 & 43 & 0.27 & 216 & & 73 & 189 & & 312 & 326 & 363 \\
\hline BP-BPA50 & 85 & 0.60 & 321 & & 148 & 324 & & 349 & 366 & 386 \\
\hline BP-BPA40 & 86 & 0.54 & 302 & & 143 & & & 353 & 374 & 391 \\
\hline BP-BPA30 & 90 & insol. & $>340^{\mathrm{d}}$ & & 90 & & & 283 & 305 & 335 \\
\hline BP-BPO70 & 77 & 0.42 & 148 & & & 150 & & 238 & 253 & 271 \\
\hline BP-BPO60 & 88 & 0.60 & 226 & 332 & 89 & 224 & 319 & 373 & 399 & 423 \\
\hline BP-BPO50 & 88 & 0.93 & 292 & $>350^{d}$ & & 316 & $>350$ & 324 & 348 & 369 \\
\hline BP-BPO40 & 78 & 0.46 & $>300^{d}$ & & 104 & $>300$ & & 257 & 273 & 290 \\
\hline BP-RES50 & 85 & 0.16 & $172\left(230^{d}\right)$ & & & $180\left(220^{d}\right)$ & & 254 & 297 & 449 \\
\hline Nap-BPO60 & 86 & 0.33 & 249 & & 92 & & & 387 & 409 & 438 \\
\hline Nap-BPO50 & 70 & 0.20 & 268 & 346 & 90 & 235 & 340 & 335 & 389 & 422 \\
\hline Nap-RES50 & 63 & 0.64 & $230^{\mathrm{d}}$ & & & $177\left(230^{d}\right)$ & & 184 & 205 & 230 \\
\hline Nap-diol6 & 94 & 0.47 & $>330^{\mathrm{d}}$ & & 98 & $330^{\mathrm{d}}$ & & 342 & 357 & 368 \\
\hline Nap-diol10 & 90 & 0.42 & 330 & & 92 & $340^{\mathrm{d}}$ & & 352 & 364 & 373 \\
\hline
\end{tabular}

${ }^{a}$ Measured at $0.5 \mathrm{dLg}^{-1}$ in $\mathrm{PhOH} / \mathrm{TCE}(6 / 4)$ at $30^{\circ} \mathrm{C}$. ${ }^{\mathrm{b}}$ Obtained by polaring microscope with a heating stage. ${ }^{\mathrm{c}} \mathrm{Obtained}$ by $\mathrm{DSC}$ at $20 \mathrm{C} \mathrm{min}^{-1}$ heating rate, the secand heating. ${ }^{\mathrm{d}}$ Decomposition temperature. ${ }^{\mathrm{e}}$ Temperatures of $5 \%, 10 \%$, and $20 \%$ weight loss measured by TGA under $\mathrm{N}_{2}$ atmosphere.

Table IV. Properties of ternary polycarbonates

\begin{tabular}{|c|c|c|c|c|c|c|c|c|c|c|}
\hline \multirow{2}{*}{ No. } & \multirow{2}{*}{$\begin{array}{c}\text { Yield } \\
\%\end{array}$} & \multirow{2}{*}{$\frac{\eta_{\mathrm{inh}}^{\mathrm{a}}}{\mathrm{dL} \mathrm{g}^{-1}}$} & \multirow{2}{*}{$T_{\mathrm{m}}^{\mathrm{b}}$} & \multirow{2}{*}{$T_{\mathrm{i}}^{\mathrm{b}}$} & \multicolumn{3}{|c|}{$\mathrm{DSC}^{\mathrm{c}}$} & \multicolumn{3}{|c|}{$\mathrm{TGA}^{\mathrm{d}}$} \\
\hline & & & & & $T_{\mathrm{g}}$ & $T_{\mathrm{m}}$ & $T_{\mathrm{i}}$ & $5 \%$ & $10 \%$ & $20 \%$ \\
\hline Nap-Me-BPO & 96 & 0.24 & 306 & & 80 & 296 & & .340 & 384 & 419 \\
\hline Nap- $t$ Bu-BPO & 81 & 0.25 & 215 & & 99 & 263 & & 337 & 388 & 423 \\
\hline Nap-Ph-BPO & 93 & 0.33 & 202 & & 100 & & & 358 & 389 & 416 \\
\hline Nap-Me-BP & 84 & 0.36 & 278 & 372 & 93 & 281 & 363 & 380 & 399 & 423 \\
\hline Nap- $t$ Bu-BP & 83 & 0.30 & 323 & & 104 & 327 & & 339 & 370 & 386 \\
\hline Nap-Ph-BP & 93 & 0.59 & 271 & & 126 & & & 363 & 381 & 407 \\
\hline Nap-BPO-BP & 96 & 0.59 & 252 & 375 & 100 & 251 & 357 & 377 & 401 & 434 \\
\hline Nap-BPK-BP & 92 & 0.30 & 314 & 369 & 96 & 297 & - & 298 & 363 & 408 \\
\hline Nap-BPS-BP & 93 & 0.32 & 314 & & 145 & 317 & & 369 & 396 & 421 \\
\hline Nap-BPA-BP & 96 & 0.66 & 266 & & 149 & 268 & & 405 & 427 & 452 \\
\hline Nap-BPSI-BP & 96 & 0.45 & 320 & & 106 & 319 & & 378 & 398 & 422 \\
\hline
\end{tabular}

${ }^{a}$ Measured at $0.5 \mathrm{dLg}^{-1}$ in $\mathrm{PhOH} / \mathrm{TCE}(6 / 4)$ at $30^{\circ} \mathrm{C}$. ${ }^{\mathrm{b}}$ Obtained by polaring microscope with a heating stage. ${ }^{\mathrm{c}}$ Obtained by DSC at $20^{\circ} \mathrm{C} \mathrm{min}^{-1}$ heating rate, the secand heating. ${ }^{\mathrm{d}}$ Temperatures of $5 \%, 10 \%$, and $20 \%$ weight loss measured by TGA under $\mathrm{N}_{2}$ atmosphere.

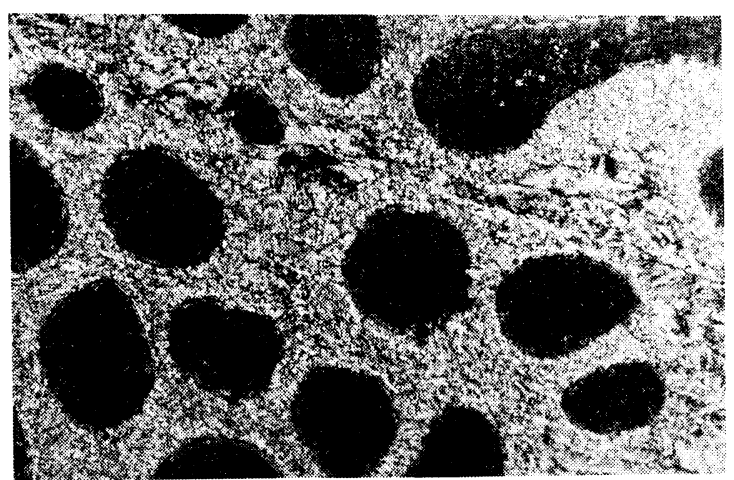

Figure 3. Nematic texture of polymer BP-BPO60 at $300^{\circ} \mathrm{C}$.

It is interesting that the non-linearity of the carbonate group was compensated for by the bent shape BPO unit, but the rigidity of polymer chain would be reduced by introducing a large amount of BPO unit.

In the third paper of this series, ${ }^{21}$ a polymer with alternating structure was synthesized by melt polyPolym. J., Vol. 29, No. 1, 1997 condensation of 4,4'-biphenyl diol and 1,3-phenylene bis(phenoxycarbonate). Excellent mesophase stability up to $400^{\circ} \mathrm{C}$ was observed. However, in this study, polymer BP-RES50 with random structure synthesized by interfacial polycondensation from 4,4'-biphenyl diol, resorcinol, and triphosgene showed poor thermal stability. By optical polarized microscope, polymer BP-RES50 melted and recrystallized at $172^{\circ} \mathrm{C}$, and decomposed at about $230^{\circ} \mathrm{C}$. These results suggest that the thermal and mesophase stability of the polycarbonates with alternating structure are better than those of the polycarbonate with random structure, because the poly(1,3-phenylenecarbonate) entity which has poor thermal stability (it decomposes at $230^{\circ} \mathrm{C}$ ) was formed in the polycarbonate BP-RES50.

Polyesters containing the 2,6-naphthalene unit, synthesized by Jackson, ${ }^{29}$ have lower melting temperature and exhibit a wider mesophase range. In this study, the influence of the structures of polycarbonate derived from 2,6-naphthalene structure and various diols on the LC properties were investigated. No liquid crystalline be- 


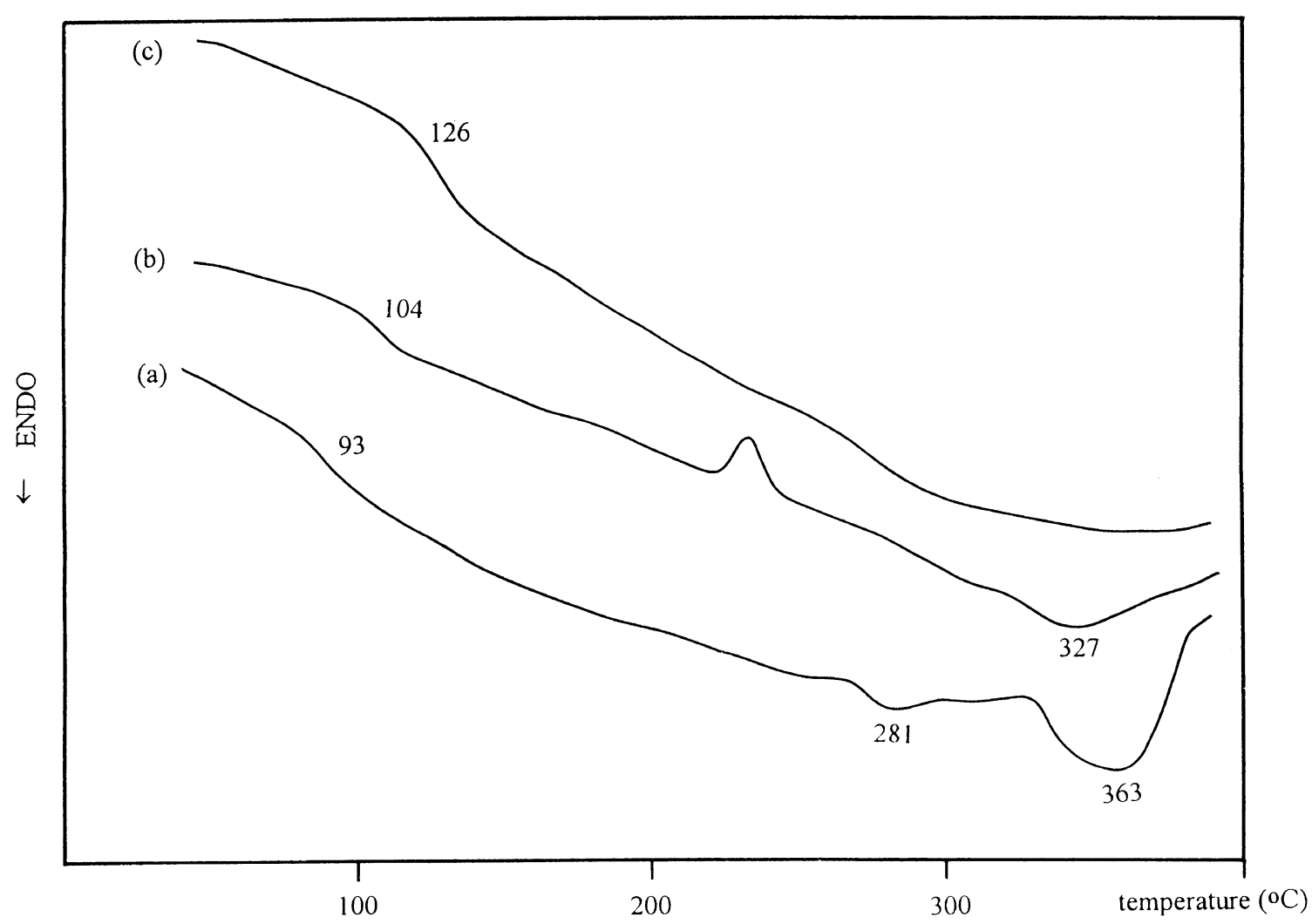

Figure 4. DSC measurements of (a) polymer Nap-Me-BP, Nap- $t \mathrm{Bu}-\mathrm{BP}$, (c) Nap-Ph-BP, at a heating rate of $20^{\circ} \mathrm{C} \min ^{-1}$.

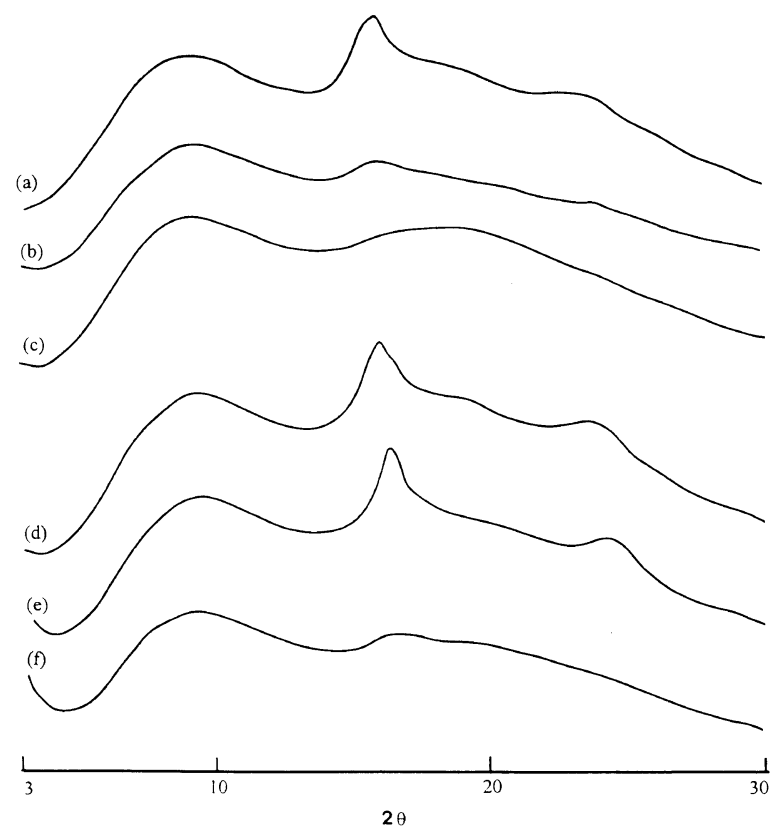

Figure 5. WAXD measurements of polymers (a) Nap-Me-BPO, (b) Nap- $t$ Bu-BPO, (c) Nap-Ph-BPO, (d) Nap-Me-BP, (e) Nap- $t$ Bu-BP, (f) $\mathrm{Nap}-\mathrm{Ph}-\mathrm{BP}$, at room temperature.

havior was found for the HQ-series polycarbonates at various BPO content. In order to examine the mesogenic character of the 2,6-naphthalene unit, polymers Nap-diol 10 and Nap-diol 6 were prepared from 2,6-naphthalenediol with 1,10-decane diol and 1,6-hexanediol, respectively. However, no LC behavior was observed for these polymers. The mesogenic character of the 2,6-nahpthalene moiety may thus be weak.

To obtain LC polycarbonates with higher molecular weight, ternary polycarbonates were synthesized from 2,6-naphthalenediol, substituted hydroquinones and BPO or BP. Bulky substituted hydroquinones ( $t$-butyl or phenyl) reduced the melting point and increased the solubility of the polymers. Thus, polymers with higher molecular weight could be obtained. However, polycarbonates synthesized from these bulky substituted hydroquinone ( $t$-butyl or phenyl) did not exhibit LC properties, possibly due to the steric effects of the substituents which force the polycarbonates to take on a non-linear conformation. With a smaller substituted hydroquinone (methyl), polymer Nap-Me-BP possesses LC properties. DSC curves of polymer Nap-Me-BP, Nap- $t \mathrm{Bu}-\mathrm{BP}$, and Nap-Ph-BP are shown in Figure 4, the DSC curve of polymer Nap-Me-BP showed a glass transition $\left(T_{\mathrm{g}}\right)$ and two endotherms corresponding to a melting transition and isotropic transition at $93^{\circ} \mathrm{C}$, $281^{\circ} \mathrm{C}$, and $363^{\circ} \mathrm{C}$, respectively. A nematic melt was found for polymer Nap-Me-BP by thermal optical polarized microscopic observation. Polymer Nap-Ph-BP shows a glass transition at $126^{\circ} \mathrm{C}$ on the DSC second heating curve. This indicates that polymer Nap-Ph-BP with bulky phenyl substituents is an amorphous material, which could also be identified by WAXD.

The X-ray diffraction patterns of ternary polycarbonates with various substituted hydroquinones at room temperature are shown in Figure 5. They indicate that polycarbonates with bulky substitutents ( $t$-butyl or phenyl) have reduced packing, and thus less crystallinity than polycarbonates with methyl substitutent. There is no significant difference in the crystallinity of polycarbonates containing BP or BPO structures.

In our previous studies on aromatic liquid crystalline polycarbonates, ${ }^{21,22}$ the non-linearity of the carbonate 


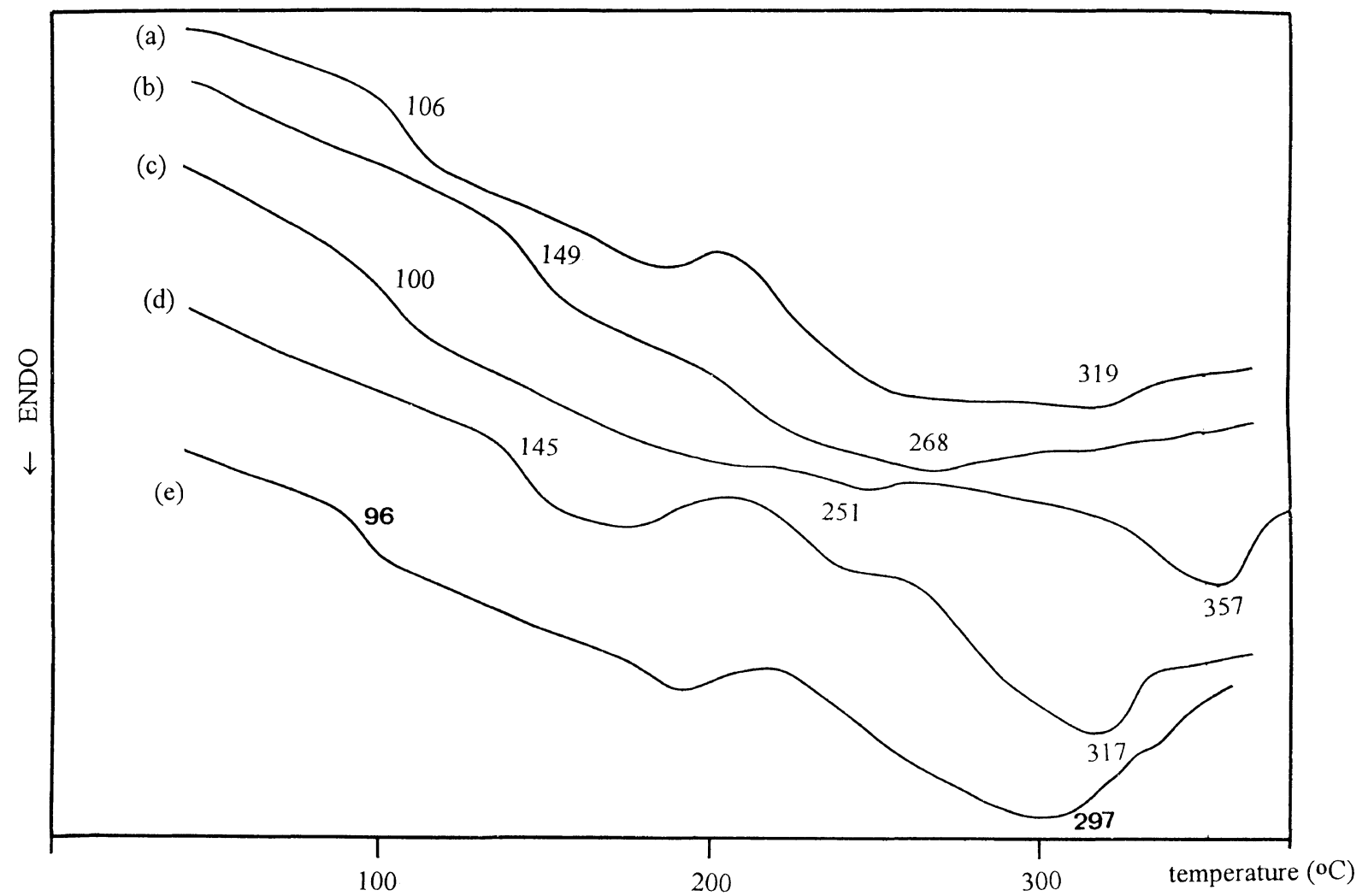

Figure 6. DSC measurements of (a) polymer Nap-BPSI-BP, (b) Nap-BPA-BP, (c) NAP-BPO-BP, (d) Nap-BPS-BP, (e) Nap-BPK-BP, at a heating rate of $20^{\circ} \mathrm{C} \mathrm{min}^{-1}$.

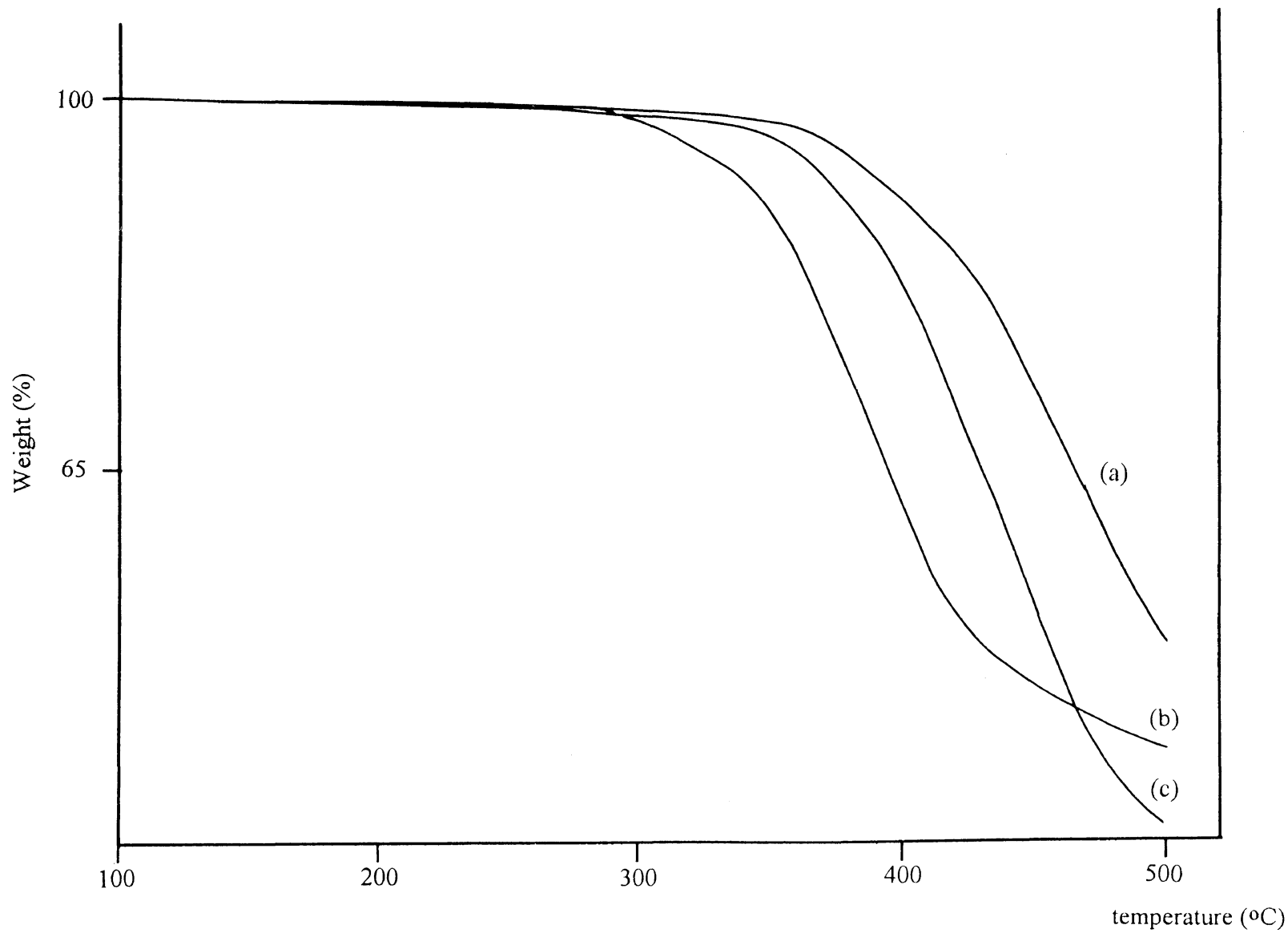

Figure 7. Thermogravimetric analysis of polymers (a) Nap-BPO-BP, (b) BP-BPO50, (c) Nap-Ph-BP. 
linkage was compensated for by the bent shape of units, such as 1,3-phenylene, 1,2-phenylene. Various aromatic polycarbonates derived from $\mathrm{BP}$ and bisphenols were reported by Kricheldorf. ${ }^{18-19}$ Polycarbonates with $50 \%$ BPO or 4,4'-dihydroxybenzophenone (BPK) possess excellent mesophase stability. The thermotropic LC properties of the polycarbonates may thus be strongly dependent on the structures of bisphenols, and LC polycarbonates not obtainable with bisphenols with bond angles $<120^{\circ}$. In this study, to investigate the effects of non-linear bisphenyl structures on the LC properties, several ternary polycabonates were synthesized from 2,6-naphthalene diol and bisphenols (BPO, BPS, BPA, PBSI, and BPK). Only Nap-BPO-BP and Nap-BPK-BP polymers possessed nematic LC properties and other polymers did not exhibit significant LC texture by thermal optical polarized microscopic observation. As shown in Figure 6, DSC curves of ternary polymers with bisphenol structures showed a glass transition and endotherm corresponding to a common melting transition. The DSC curve of polymer Nap-BPO-BP showed a glass transition and two endotherms corresponding to melting transition and isotropic transition at $100^{\circ} \mathrm{C}$, $251^{\circ} \mathrm{C}$, and $357^{\circ} \mathrm{C}$, respectively.

Thermogravimetric analysis data of the synthesized polymers are shown in Tables III and IV. Typical TGA curves of polycarbonates Nap-BPO-BP, BP-BPO50, and Nap-Ph-BP are shown in Figure 7. Most of the synthesized polycarbonates possessed good thermal stability. However, polycarbonates derived from resorcinol show poor thermal stability. Except for Nap-RES50, no significant differences in the thermal stability of NAPseries polycarbonates derived from various bisphenols or hydroquinones (methyl, $t$-butyl, or phenyl) could be found.

Acknowledgment. The authors are indebted to the National Science Council of Republic of China for financial support of this work (NSC-84-2216-E-033-006).

\section{REFERENCES}

1. P. K. Bhowmik and R. W. Lenz, J. Polym. Sci. Polym. Chem., 31, 2115 (1993).

2. R. O. Garay, P. K. Bhowmik, and R. W. Lenz, J. Polym. Sci., Polym. Chem., 31, 1001 (1993).

3. F. Navarro and J. L. Serrano, J. Polym. Sci., Polym. Chem., 30, 1789 (1992).

4. C. H. Li, W. W. Lai, K. Y. Hsu, and T. C. Chang, J. Polym. Sci., Polym. Chem., 31, 27 (1993).

5. H. R. Kricheldorf and R. Huner, Makromol. Chem., Rapid Commun., 11, 211 (1990).

6. H. R. Kricheldorf, R. Pakull, and S. Buchner, J. Polym. Sci., Polym. Chem., 27, 431 (1989).

7. H. R. Kricheldorf and R. Pakull, Polymer, 28, 1772 (1987).

8. H. R. Kricheldorf, R. Pakull, and S. Buchner, Mecromolecules, 21, 1929 (1988).

9. S. M. Aharoni, Mecromolecules, 22, 686 (1989).

10. C. H. Li, K. Y. Hsu, and T. C. Chang, J. Polym. Sci., Polym. Chem., 31, 1119 (1993).

11. C. H. Li, H. P. Cheng, T. C. Chang, and T. Y. Chu, J. Polym. Sci., Polym. Chem., 31, 1125 (1993).

12. C. H. Li and T. C. Chang, J. Polym. Sci., Polym. Chem., 28, 3625 (1990).

13. W. Mormann and M. Brahm, Makromol. Chem., 190, 631 (1989).

14. M. Tanaka and T. Nakaya, Makromol. Chem., 187, 2345 (1986).

15. Y. C. Lai, B. T. Debona, and D. C. Prevorsek, J. Appl. Polym. Sci., 36, 819 (1988).

16. H. K. Mahabada and L. Alexandru, U. S. Patent, 4,543,313.

17. D. N. Schissel, U. S. Patent, $4,831,105$.

18. H. R. Kricheldorf and D. Lubbers, Makromol. Chem., Rapid Commun., 10, 383 (1989).

19. H. R. Kricheldorf and D. Lubbers, Macromolecules, 23, 2656 (1990).

20. T. Hirata, M. Sato, and K. Mukaida, Makromol. Chem., 194, 2861 (1993).

21. S. J. Sun and T. C. Chang, J. Polym. Sci., Polym. Chem., 31, 2237 (1993).

22. S. J. Sun and T. C. Chang, J. Polym. Sci., Polym. Chem., 31, 2711 (1993).

23. H. Schnell, "Chemistry and Physics of Polycarbonate," New York, N.Y., (1964).

24. T. Kanno and M. Ueda, Makromol. Chem., Rapid Commun, 11, 181 (1990).

25. Y. Saegusa, M. Kuriki, A. Kawai, and S. Nakamura, J. Polym. Sci., Polym. Chem., 28, 3327 (1990).

26. D. J. Brunelle and T. G. Shannon, Macromolecules, 24, 3035 (1991).

27. S. Oi, K. Nemoto, S. Matsuno, and Y. Inoue, Makromol. Chem., Rapid Commun., 15, 133 (1994).

28. R. W. Lenz and J. I. Jin, Macromolecules, 14, 1405 (1981).

29. W. J. Jackson, Macromolecules, 16, 1027 (1983). 